\title{
Relationship between calcific tendinopathy and rotator cuff tear on shoulder magnetic resonance imaging: case-controlled comparison
}

\author{
Hamza Sucuoğlu ${ }^{1 A, B, C, D, E, F, G}$, Abdülkadir Asan²A,B,C,D,E,F,G \\ 'School of Health Sciences, Istanbul Gelisim University, Istanbul, Turkey \\ 2Department of Radiology, Private Nisa Hospital, Istanbul, Turkey
}

\section{Abstract}

Purpose: To determine the frequency of rotator cuff tear on the shoulder magnetic resonance imaging (MRI) of patients with rotator cuff calcific tendinopathy and the relationship between rotator cuff tear and calcific tendinopathy.

Material and methods: In this retrospective case control study, 137 patients with calcific tendinopathy and 137 control group patients without calcific tendinopathy with shoulder pain, whose age, sex, and shoulder laterality values were matched, were compared in terms of rotator cuff tears on their shoulder MRI images.

Results: The frequency of rotator cuff tear was found to be significantly higher in the control group (37.2\%) compared to the calcific tendinopathy group $(23.4 \%)(p<0.01)$. Partial thickness was $81.3 \%$ in the calcific tendinopathy group and $70.6 \%$ in the control group, and no significant difference was observed between the two groups in terms of the size of the rotator cuff tear $(p>0.05)$. In the calcific tendinopathy group, there was no significant relationship between the localisation of calcification and the rotator cuff tear, and only in $4.4 \%$ of the participants were calcification and tear at the same location on the same tendon $(p>0.05, r=0.04)$.

Conclusions: The patients with calcific tendinopathy, who had been admitted with shoulder pain, did not demonstrate an increased risk of rotator cuff tear based on their MRI compared to patients with shoulder pain without calcific tendinopathy. No significant relationship was determined between calcific tendinopathy and rotator cuff tear.

Key words: shoulder, calcific tendinopathy, rotator cuff tear, musculoskeletal system, hydroxyapatite, magnetic resonance imaging.

\section{Introduction}

Calcific tendinopathy $(\mathrm{CT})$ is a disease, the cause of which is not known, and it is characterised by hydroxyapatite accumulation in the rotator cuff tendon, muscle, or the subacromial-subdeltoid bursa when calcification spreads around the tendons [1]. CT, which is frequently encountered in middle-aged patients, is a common asymptomatic finding on imaging studies, which accounts for shoulder pain in approximately $7 \%$ of cases [2]. It has been reported in the radiological (magnetic resonance imaging [MRI] or ultrasound) examinations of symptomatic or asymptomatic shoulders that the prevalence of rotator cuff tendon tears (RCT) varies between $30 \%$ and 50\% [3].

CT and RCT can mimic each other with similar clinical features at presentation and can be seen together in the same patient [2]. This makes it difficult to differentiate between these two disorders, which have different treatment approaches. Furthermore, it is still controversial that CT and RCT tend to provide the background for the formation of each other and that they play a role in the aetiopathogenesis of each other [4-6]. In the literature, studies investigating the relationship between CT and RCT are usually based on surgical and arthrographic findings.

Correspondence address:

Dr. Hamza Sucuoğlu, School of Health Sciences, Istanbul Gelisim University, Istanbul, Turkey, e-mail: hamzasucuoglu@gmail.com

Authors' contribution:

A Study design · B Data collection · C Statistical analysis · D Data interpretation · E Manuscript preparation · F Literature search · G Funds collection 
These studies reported that $28 \%$ of patients with CT had RCT and $23 \%$ of patients with RCT had CT $[7,8]$. Another operative study reported that RCT occurred rarely due to an underlying CT [9]. Although surgical intervention is rarely required in $\mathrm{CT}$, the fact that these studies are based on surgical findings, as well as the contradictory results, increases the probability of bias. In this context, interpretation of the relationship between CT and RCT according to the results of these studies would not be reliable [4]. For all these reasons, further studies are needed to clarify the frequency of co-occurrence of CT and RCT together with the causal relationship between them. Radiological evaluation with shoulder MRI is a good option because it will provide more accurate results [10]. To the best of our knowledge, there is only one study in the literature investigating the relationship between CT and RCT with MRI. In this study, there was no significant difference in the incidence of RCT between the CT group and the controls [4].

We wonder whether the difference would be significant if the study power were larger. For this purpose, we aimed to determine the frequency of RCT in the shoulder MRIs of CT patients and the relationship between CT and RCT in this study with more participants.

\section{Material and methods}

\section{Participants and examinations}

The study was approved by the Ethics Board of Istanbul Gelisim University (protocol no: 2018-20-12). The reports of the shoulder MRIs carried out on patients who had been admitted with complaints of shoulder pain in Private Nisa Hospital in the period between May 2014 and August 2018 were examined retrospectively. CT was mentioned in 155 (5.1\%) out of the 3028 shoulder MRI reports in total (Figure 1). These reports about CT and their MRIs were reviewed again by a radiologist with 15 years of experi-

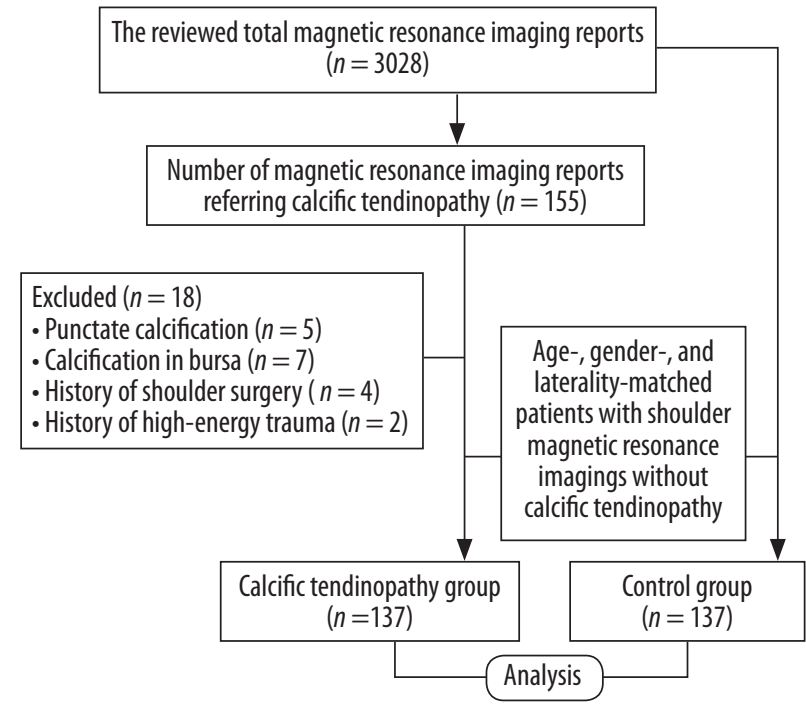

Figure 1. Study flow chart ence regarding the musculoskeletal system. In all the MRI examinations, fluid-sensitive sequences (with fat-suppressed proton density, T1 weight, and gradient echo T2 weight) were used in three planes (sagittal oblique, coronal oblique, and axial) for defining and measuring the rotator cuff calcifications and tears. CT was defined with homogenous or heterogeneous, low signal, round hypointensities suggestive of calcium accumulations in all the sequences of the MRI. Punctate calcifications were considered to be dystrophic calcifications, and these were not included in the study. In the MRIs, the location of $\mathrm{CT}$ and its relationship with the tendon were examined. Calcification of the tendons was recorded as interstitially settled, articular-sided, or bursa-sided. Cases with calcifications not located within the rotator cuff tendons were excluded from the study. In cases with calcification in more than one tendon, the tendon covering the largest calcification focus was used for the analysis. Moreover, the patients who had previous shoulder surgery findings on the MRI report or those who were determined to have had a history of high-energy trauma in their medical records were excluded. In conjunction with these criteria, 137 participants remained in the CT group because 18 out of the 155 shoulder MRI reports indicating CT were excluded from the study (Figure 1).

The rotator cuff tendons of the patients with CT on their MRI reports were assessed in terms of tears. In the case of presence of RCT, the tendon and size (partial- or full-thickness tear) and its relationship with the localisation of calcification (different tendons, same tendon-different location, and same tendon-same location) were recorded. Full-thickness rotator cuff tears are a type of rotator cuff tear that extend from the bursal surface to the articular surface. Partial thickness means that the rotator cuff tear is located only on the bursal surface or joint surface or is intratendinous. Moreover, the medical records of each patient were obtained, and information regarding their age, sex, and comorbidities (diabetes mellitus, hypothyroidism, inflammatory/crystalline arthritis, connective tissue disease) was recorded.

The control group was formed with 137 patients who did not have CT and who had shoulder MRIs in which the shoulder laterality was matched individually with the CT group (Figure 1). Accordingly, each control group patient was matched with a CT patient with the same age, sex, and shoulder laterality. In the control group, with a similar process to that in the CT group, the RCT presence, location, and size were assessed in the MRIs of the patients, and their comorbidities were recorded from their medical records.

Shoulder radiographies - if there were any - performed in the symptomatic period of the patients and within 30 days of their MRI were reviewed retrospectively. Shoulder radiography images were available in 40 (29.2\%) of the patients in the CT group, and calcification corresponding to CT defined in MRI was observed in all of these radiography images. 


\section{Statistical analyses}

All statistical analyses were performed using the SPSS software (ver. 22.0; SPSS, Inc., Chicago, IL). The odds ratio and its $95 \%$ confidence interval (CI) of having rotator cuff tear in the cases of calcific tendinopathy were calculated for the comparisons between the groups. The calcific tendinopathy group and the control group were compared based on their variables of age, sex, comorbidities (diabetes mellitus, hypothyroidism, inflammatory/crystalline arthritis, connective tissue disease), shoulder laterality (left, right), rotator cuff tear incidence (no, yes), rotator cuff tear size (full and partial thickness), and rotator cuff tear location (supraspinatus, infraspinatus, subscapularis). These comparisons were made using the paired-samples $t$-test for the continuous variables and the $\chi^{2}$ test (or Fischer's exact test) for the categorical variables. The location and distribution of calcific tendinopathy were calculated in frequencies and percentages, and their relationship with rotator cuff tear was assessed with the Spearman's rho parametric correlation test.

\section{Results}

Because the control group was formed by matching age, sex, and shoulder laterality with the participants in the CT group, there was no significant difference between the groups in terms of age (mean age 52.6 years), sex (72.3\% female), or shoulder side (55.5\% right) $(p=1.0)$ (Table 1). While the incidence rates of diabetes and hypothyroidism were higher in the CT group, only the higher incidence of diabetes was statistically significant $(p<0.05)$. In terms of the incidence rates of other comorbidities such as arthritis and connective tissue disease, there was no significant difference between the groups (Table 1).

The RCT incidence was found to be significantly higher in the control group (37.2\%) in comparison to the CT group $(23.4 \%)(p<0.05)$ (Table 1). There was no significant difference between the groups in terms of the size of RCT, and partial thickness was more prevalent in both groups $(p=0.31)$ (Table 1$)$.

In the CT group, $68.8 \%$ of the RCT cases were localised in the supraspinatus tendon, while in the control group, $78.5 \%$

Table 1. Demographics and tear incidence comparison between calcific tendinopathy patients and the control group

\begin{tabular}{|c|c|c|c|c|}
\hline Variable & Total $(n=274)$ & Calcific tendinopathy group ( $n=137$ ) & Control group ( $n=137)$ & $p$ value \\
\hline Age (mean $\pm S D)$ & $52.6 \pm 11.8$ & $52.6 \pm 11.9$ & $52.6 \pm 11.8$ & $0.98^{*}$ \\
\hline \multicolumn{5}{|l|}{ Sex, $n(\%)$} \\
\hline Female & $197(71.9)$ & $99(72.3)$ & $99(72.3)$ & \multirow[t]{2}{*}{$1.0^{* *}$} \\
\hline Male & $77(28.1)$ & $38(27.7)$ & $38(27.7)$ & \\
\hline \multicolumn{5}{|l|}{ Comorbidities, $n(\%)$} \\
\hline Diabetes mellitus & $44(16)$ & $29(21.2)$ & $15(10.9)$ & $0.02^{* *}$ \\
\hline Hypothyroidism & $16(5.8)$ & $11(8)$ & $5(3.6)$ & $0.12^{* *}$ \\
\hline Inflammatory/crystalline arthritis & $9(3.2)$ & $5(3.6)$ & $4(2.9)$ & $1.0^{* * *}$ \\
\hline Connective tissue disease & $2(0.7)$ & $1(0.7)$ & $1(0.7)$ & $1.0^{* * *}$ \\
\hline \multicolumn{5}{|l|}{ Shoulder side, $n(\%)$} \\
\hline Left & $120(43.8)$ & $61(44.5)$ & $59(43.1)$ & \multirow[t]{2}{*}{$0.88^{* *}$} \\
\hline Right & $154(56.2)$ & $76(55.5)$ & $78(56.9)$ & \\
\hline \multicolumn{5}{|l|}{ Rotator cuff tear, $n(\%)$} \\
\hline No & $191(69.7)$ & $105(76.6)$ & $86(62.8)$ & \multirow[t]{2}{*}{$0.01^{* *}$} \\
\hline Yes & $83(30.3)$ & $32(23.4)$ & $51(37.2)$ & \\
\hline \multicolumn{5}{|l|}{ Rotator cuff tear size, $n(\%)$} \\
\hline Full thickness & $21(25.3)$ & $6(18.7)$ & $15(29.4)$ & \multirow[t]{2}{*}{$0.31^{* * *}$} \\
\hline Partial thickness & 62 (74.7) & $26(81.3)$ & $36(70.6)$ & \\
\hline \multicolumn{5}{|l|}{ Rotator cuff tear location, $n(\%)$} \\
\hline Supraspinatus & $62(74.7)$ & $22(68.8)$ & $40(78.5)$ & \multirow[t]{3}{*}{$0.13^{* *}$} \\
\hline Infraspinatus & $6(7.2)$ & $4(12.5)$ & $2(3.9)$ & \\
\hline Subscapularis & $15(18.1)$ & $6(18.7)$ & $9(17.6)$ & \\
\hline
\end{tabular}

*Denotes $p$ values obtained using a two-sample $t$-test

${ }^{* *}$ Denotes $p$-values obtained using the $\chi^{2}$ test

***Denotes $p$-values obtained using Fisher's exact test 


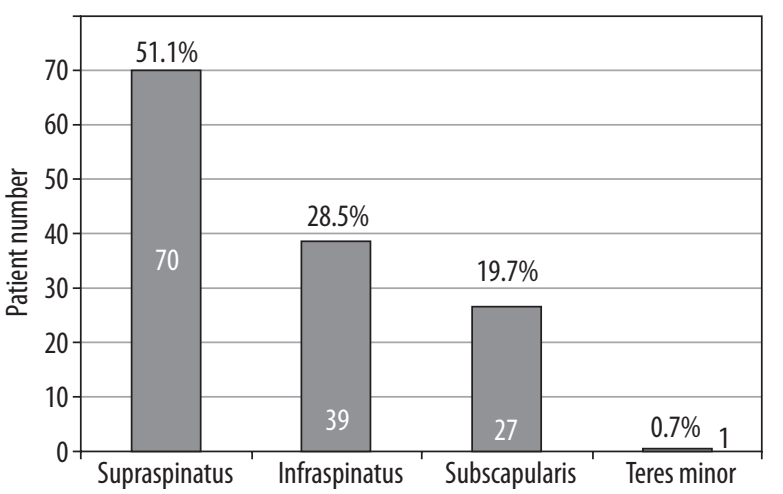

Figure 2. Location and distribution of calcific tendinopathy in rotator cuff tendons

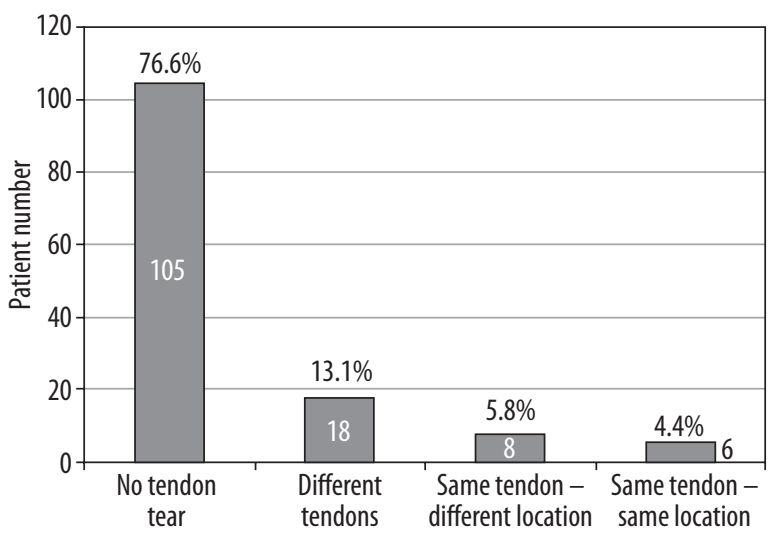

Figure 3. Correlation between the rotator cuff tear and tendon calcification location in patients with calcific tendinopathy

*Spearman's rho nonparametric correlation test $(p=0.58, r=0.04)$

of these were localised in the supraspinatus tendon. Nevertheless, there was no significant difference in terms of the location of RCT between the two groups $(p=0.13)$ (Table 1).

It was observed that calcification was most frequent in the supraspinatus (51.1\%) and infraspinatus (28.5\%) tendons. The frequency of calcification was less in subscapularis (19.7\%) and teres minor (0.7\%) tendons (Figure 2).

In addition, seven patients had calcification in more than one tendon, four of them had smaller teres minor tendons, and three of them had subscapularis tendons.

In the CT group, there was no significant relationship between the localisation of calcification and RCT $(p=0.58$, $r=0.04)$. In $18(13.1 \%)$ of all the patients with CT, there was a tear in a tendon other than the tendon where calcification was present, and in only six (4.4\%) were the calcification and tear at the same location in the same tendon (Figure 3).

\section{Discussion}

The relationship between calcific tendinopathy and rotator cuff tears has been a subject of debate for a long time. There are four debated ideas in determining the relationship between CT and RCT. These are: the pathogeneses of CT and RCT are similar; CT develops as a result of RCT; CT initiates the formation of RCT; and there is no relationship between CT and RCT [4].

In the pathogenesis of $\mathrm{CT}$, the main factor is hydroxyapatite accumulation with calcium phosphate content in the rotator cuff tendons. It has been argued for a long time that this accumulation, the exact aetiology of which is unknown, arises from degeneration in tendon fibres and focal necrosis $[11,12]$. In later studies, it was reported that metaplasia in tendon fibres [13], mis-differentiation of tendon-derived stem cells to chondrocytes and osteoblasts [14], endocrine disorders (thyroxine, oestrogen, insulin) [15,16], and genetic factors [17-19] may also be related to the disease development. The incidence rates of diabetes and hypothyroidism, which have a known relationship with CT, were also high in our study, but this high degree was statistically significant only for diabetes. In all the aforementioned cases, even if it could be estimated that the development of CT may be higher, required further clarification of the pathogenesis of CT.

RCT aetiopathogenesis was classified as extrinsic or intrinsic factors. Extrinsic factors comprise trauma, excessive use, chronic impingement, and multifactorial conditions (nicotine, diabetes, obesity etc.), which may increase the tissue damage. Intrinsic factors are listed as hypoperfusion, ageing or degeneration in the tendon, apoptotic theory, extra cellular matrix modifications, and calcification [20,21].

RCT occurs most frequently as a result of tendon degeneration $[4,20]$. These degenerations and tears in the supraspinatus and infraspinatus tendons are frequently observed in the area defined as "the avascular critical zone", the congestion of which has decreased relatively. This area has also been defined as a commonly observed location of rotator cuff calcification $[5,6]$. Dystrophic calcification of the degenerated tendon is also frequently seen in this region [21]. For this reason, some authors believe that CT is related to the degenerative tear of the rotator cuff $[5,6]$. In contrast, in our study, a significant portion of the RCT cases observed in CT were observed in a different tendon or a different location in the same tendon. Moreover, findings regarding whether or not CT is the premise of degenerative tendon tear differ [22,23]. Clinically, while CT is observed more frequently in middle-aged individuals, the frequency of degenerative tears increases along with increased age $[24,25]$. CT may be observed not only in the supraspinatus and infraspinatus tendons with the avascular critical zone, but also in the subscapularis and teres minor tendons without the avascular zone [5]. The findings of our study supported this, and there was also calcification in the subscapularis (19.7\%) and teres minor (0.7\%) tendons of patients with CT. In our study, the fact that calcification and rupture can be in a different tendon or at a different location, and that calcification can be seen in tendons without an avascular zone, suggests that CT has a different aetiopathogenesis than tendon degeneration. 
In addition, dystrophic calcifications in the degenerated rotator cuff tendon have some radiological features that distinguish them from calcific deposits in CT. Dystrophic calcifications are generally smaller than $5 \mathrm{~mm}$ in size, adjacent to tuberosity, and they are more dense and solid [26]. In our study, we did not measure the calcification dimensions and did not consider punctate calcifications as dystrophic, and hence did not include them. However, the intensity and hardness of calcifications could not be evaluated with MRI.

There are some studies alleging that CT may increase the tendency of development of RCT $[7,10]$. Merolla et al. [10] stated that previous abnormal calcifications may generate or increase RCT at a level that may require surgical treatment. Likewise, Jim et al. [7] reported that there was partial- or full-thickness RCT in $27 \%$ of the 81 patients with CT in whom arthrography had been performed, small calcifications more frequently accompanied RCT, and the comorbidity of these two disorders was more frequent in elderly people. Some operation-related studies have found that RCT has rarely occurred only in the presence of CT [9], and there are also studies showing that calcification occurred in the tendons in $23 \%$ of RCT patients [8]. Considering that these studies are based on operational findings, and only patients resistant to conservative treatment would proceed to surgical intervention, it would not be completely accurate to state that CT and RCT are related to each other. For this reason, MRI will be more objective and useful for evaluating the relationship between CT or RCT or association for all patients with shoulder pain [10,26]. On MRI, calcific accumulation has a low signal intensity. However, the accuracy of MRI in determining calcific accumulation is about 95\%, although areas around it, such as increased payout-related signal intensity, may be misinterpreted as an RCT [10,26-29]. In addition, Nörenberg et al. [30] showed that susceptibility-weighted MRI had 98\% sensitivity and $96 \%$ specificity in the identification of shoulder calcifications compared to radiography. Likewise, we also used MRI in our study.

In some imaging studies, it has been demonstrated that CT is not related to development of RCT. In an ultrasonography study, RCT related to the calcification area was not determined in $94 \mathrm{CT}$ patients, whose mean age was 57 years [31]. Beckmann et al. [4] examined the shoulder MRIs of 86 CT patients and 86 control patients with shoulder pain, who were matched with the former group, and reported that the difference in the RCT incidence rates - that they determined as $27.9 \%$ in the CT group and $34.9 \%$ in the control group - was not statistically significant. Moreover, in the CT group, in only three out of eight patients (3.5\% of all CT) - with calcification and RCT on the same tendon - the calcification and tear were determined at the same location of the tendon. Additionally, $87.5 \%$ of the tears in the CT group had partial thickness, $63.3 \%$ of the tears in the control group had full thickness, and the sizes of RCT were found to be significantly different [4]. Unlike Beckman et al. [4], there was significantly less RCT in the CT group in our study. This may be due to the higher incidence of RCT in patients presenting with shoulder pain than CT incidence. But we cannot talk about a certain reason. Furthermore, no significant difference was found between the groups in terms of the RCT size. Only $4.4 \%$ of all patients in the CT group had calcifications and tears at the same location of the same tendon. All these results in our study supported the idea that CT does not develop as a result of RCT, and it does not result in a tendency towards RCT.

Similar to the studies in the literature $[4,32,33]$, CT and RCT were most frequently observed in the supraspinatus tendon in our study, and this tendon was followed by the infraspinatus and subscapularis tendons.

\section{Limitations}

Our study had some limitations. In this study, an analysis and correlation between MRI findings such as shoulder joint instability, degree of subacromial stenosis and clinical conditions such as ROM limitation, and duration and degree of pain were not evaluated. Our study evaluated the current and static relationship of CT with the tendon. It did not assess whether CT increased the risk of faster tendon degeneration and tendon tears over a longer period of time. In the study, surgical or MR arthrography - which may be more sensitive and specific - were not used in the diagnosis of rotator cuff tear, but only MRI was used. This may have caused us to overlook the micro-tears in the tendons. Another limitation was the difficulty in distinguishing CT from dystrophic calcification in the degenerated tendon by MRI. However, punctate calcifications with a small size were considered dystrophic and hence were excluded from the study. Moreover, the diagnosis of CT was made based on the MRIs without verifying the presence of calcification on the radiographies. However, all CT patients with radiographs showed calcifications on radiographs.

\section{Conclusions}

Patients with calcific tendinopathy, who presented to the hospital with complaints of shoulder pain, did not have more rotator cuff tears in the MRI reports in comparison to patients with shoulder pain without calcific tendinopathy. No significant relationship was found between calcific tendinopathy and rotator cuff tear. These two conditions probably arise due to different aetiopathogenetic factors.

\section{Acknowledgements}

Thank you to M.D. Adem Akturk, the Global Translation and the Medical Translation Companies (Istanbul, Turkey) for professional language translation service.

\section{Conflicts of interest}

The authors declare that they have no conflicts of interest. 


\section{References}

1. Komurcu M, Kilic E. Calcifying tendinitis: etiology, pathogenesis and treatment methods. Turkiye Klinikleri J Surg Med Sci 2007; 3: 17-21.

2. Greis AC, Derrington SM, McAuliffe M. Evaluation and nonsurgical management of rotator cuff calcific tendinopathy. Orthop Clin North Am 2015; 46: 293-302.

3. Reilly P, Macleod I, Macfarlane R, et al. Dead men and radiologists don't lie: a review of cadaveric and radiological studies of rotator cuff tear prevalence. Ann R Coll Surg Engl 2006; 88: 116-21.

4. Beckmann NM, Tran MQ, Cai C. Incidence of rotator cuff tears in the setting of calcific tendinopathy on MRI: a case controlled comparison. Skeletal Radiol 2019; 48: 245-250.

5. Rathbun JB, Macnab I. The microvascular pattern of the rotator cuff. J Bone Joint Surg Br 1970; 52: 540-553.

6. Moseley HF, Goldie I. The arterial pattern of the rotator cuff of the shoulder. J Bone Joint Surg 1963; 48: 780.

7. Jim YF, Hsu HC, Chang CY, Wu JJ, Chang T. Coexistence of calcific tendinitis and rotator cuff tear: an arthrographic study. Skeletal Radiol 1993; 22: 183-185.

8. Wolfgang GL. Surgical repair of tears of the rotator cuff of the shoulder: factors influencing the result. J Bone Joint Surg Am 1974; 56: 14-26.

9. McLaughlin HL, Asherman EG. Lesions of the musculotendinous cuff of the shoulder. IV. Some observations based upon the results of surgical repair. J Bone Joint Surg Am 1951; 33: 76-86.

10. Merolla G, Singh S, Paladini P, Porcellini G. Calcific tendinitis of the rotator cuff: state of the art in diagnosis and treatment. J Orthopaed Traumatol 2016; 17: 7-14.

11. Codman EA. The Shoulder: Rupture of the Supraspinatus Tendon and Other Lesions in or about the Subacromial Bursa. Thomas Todd Co., Boston 1934.

12. Mohr W, Bilger S. Basic morphologic structures of calcified tendinopathy and their significance for pathogenesis. Z Rheumatol 1990; 49: 346-355.

13. Uhthoff HK, Sarkar K, Maynard JA. Calcifying tendinitis: a new concept of its pathogenesis. Clin Orthop Relat Res 1976; 118: 164-168.

14. Rui YF, Lui PP, Chan LS, et al. Does erroneous differentiation of tendon-derived stem cells contribute to the pathogenesis of calcifying tendinopathy? Chin Med J 2011; 124: 606-610.

15. Harvie P, Pollard TC, Carr AJ. Calcific tendinitis: natural history and association with endocrine disorders. J Shoulder Elbow Surg 2007; 16: 169-173.

16. Mavrikakis ME, Drimis S, Kontoyannis DA, et al. Calcific shoulder periarthritis (tendinitis) in adult onset diabetes mellitus: a controlled study. Ann Rheum Dis 1989; 48: 211-214.

17. Zhang Y, Johnson K, Russell RG, et al. Association of sporadic chondrocalcinosis with a-4-basepair G-to-A transition in the 50-untranslated region of $\mathrm{ANKH}$ that promotes enhanced expression of ANKH protein and excess generation of extracellular inorganic pyrophosphate. Arthritis Rheum 2005; 52: 1110-1117.

18. Sengar DP, McKendry RJ, Uhthoff HK. Increased frequency of HLA-A1 in calcifying tendinitis. Tissue Antigens 1987; 29: 173-174.

19. Oliva F, Barisani D, Grasso A, Maffulli N. Gene expression analysis in calcific tendinopathy of the rotator cuff. Eur Cell Mater 2011; 21: 548-557.
20. Via AG, De Cupis M, Spoliti M, Oliva F. Clinical and biological aspects of rotator cuff tears. Muscles Ligaments Tendons J 2013; 3: 70-79.

21. ElShewy MT. Calcific tendinitis of the rotator cuff. World J Orthop 2016; 7: 55-60.

22. Jozsa L, Balint BJ, Reffy A. Calcifying tendinopathy. Arch Orthop Trauma Surg 1980; 97: 305-307.

23. Kernwein GA. Roentgenographic diagnosis of shoulder dysfunction. JAMA 1965; 194: 179-183.

24. Sakamoto K, Kozuki K. Calcific tendinitis at the biceps brachii insertion of a child: a case report. J Shoulder Elbow Surg 2002; 11: 88-91.

25. Sher JS, Uribe JW, Posada A, et al. Abnormal findings on magnetic resonance images of asymptomatic shoulders. J Bone Joint Surg Am 1995; 77: 10-15.

26. Gartsman GM. Calcific tendinitis. In: Shoulder Arthroscopy. $2^{\text {nd }}$ ed. Saunders Elsevier, Philadelphia 2009; 316-321.

27. Porcellini G, Paladini P, Campi F, Pegreffi F. Osteolytic lesion of greater tuberosity in calcific tendinitis of the shoulder. J Shoulder Elbow Surg 2009; 18: 210-215.

28. Maier M, Stäbler A, Schmitz C, et al. On the impact of calcified deposits within the rotator cuff tendons in shoulders of patients with shoulder pain and dysfunction. Arch Orthop Trauma Surg 2001; 121: 371-378.

29. Chan R, Kim D, Millet P, Weissman BN. Calcifying tendinitis of rotator cuff with cortical bone erosion. Skeletal Radiol 2004; 33: 596-599.

30. Norenberg D, Ebersbergere HU, Walter T, et al. Diagnosis of calcific tendonitis of the rotator cuff by using susceptibility-weighted MR imaging. Radiology 2016; 278: 475-484.

31. Chiou HJ, Chou YH, Wu JJ, Hsu CC. Evaluation of calcific tendonitis of the rotator cuff: role of color doppler ultrasonography. J Ultrasound Med 2002; 21: 289-295.

32. Clavert P, Sirveaux F. Shoulder calcifying tendinitis. Rev Chir Orthop Reparatrice Appar Mot 2008; 94 (8 suppl): 336-355.

33. Rhee YG, Kim YH, Park MS. Arthroscopic treatment in calcific tendinitis of the shoulder. J Korean Shoulder Elbow Soc 2000; 3: 68-74. 